Int. J. Dev. Biol. 60: 297-304 (2016)

doi: $10.1387 /$ ijdb.160139oh

\title{
Single-molecule, antibody-free fluorescent visualisation of replication tracts along barcoded DNA molecules
}

\author{
FRANCESCO DE CARLI ${ }^{1,2}$, VINCENT GAGGIOLI ${ }^{1,3}$, GAËL A. MILLOT ${ }^{4,5}$ and OLIVIER HYRIEN ${ }^{*, 1}$ \\ ${ }^{1}$ Institut de Biologie de l'Ecole Normale Supérieure (IBENS), CNRS UMR 8197, Inserm U1024, Ecole Normale \\ Supérieure, PSL Research University, Paris, France, ${ }^{2}$ Sorbonne Universités, UPMC Univ Paris 06, IFD, Paris, \\ France, ${ }^{3}$ Welcome Trust/Cancer Research UK Gurdon Institute, University of Cambridge, England, UK, ${ }^{4}$ Institut \\ Curie, PSL Research University, CNRS, UMR 3244, Paris, France and ${ }^{5}$ Sorbonne Universités, UPMC Univ Paris 06, \\ CNRS, UMR 3244, Paris, France.
}

\begin{abstract}
DNA combing is a standard technique to map DNA replication at the single molecule level. Typically, replicating DNA is metabolically labelled with nucleoside or nucleotide analogs, purified, stretched on coverslips and treated with fluorescent antibodies to reveal tracts of newly synthesized DNA. Fibres containing a locus of interest can then be identified by fluorescent in situ hybridization (FISH) with DNA probes. These steps are complex and the throughput is low. Here, we describe a simpler, antibody-free method to reveal replication tracts and identify the locus of origin of combed DNA replication intermediates. DNA was replicated in Xenopus egg extracts in the presence of a fluorescent dUTP. Purified DNA was barcoded by nicking with Nt.BspOI, a sitespecific nicking endonuclease (NE), followed by limited nick-translation in the presence of another fluorescent dUTP. DNA was then stained with YOYO-1, a fluorescent DNA intercalator, and combed. Direct epifluorescence revealed the DNA molecules, their replication tracts and their Nt.BspOI sites in three distinct colours. Replication intermediates could thus be aligned to a reference genome map. In addition, replicated DNA segments showed a stronger YOYO-1 fluorescence than unreplicated segments. The entire length, replication tracts, and NE sites of combed DNA molecules can be simultaneously visualized in three distinct colours by standard epifluorescence microscopy, with no need for antibody staining and/or FISH detection. Furthermore, replication bubbles can be detected by quantitative YOYO-1 staining, eliminating the need for metabolic labelling. These results provide a starting point for genome-wide, single-molecule mapping of DNA replication in any organism.
\end{abstract}

KEY WORDS: DNA replication, DNA combing, DNA barcoding, single-molecule analysis, Xenopus egg extract

\section{Introduction}

Since their first use four decades ago (Gandini Attardi et al., 1976, Mattoccia et al., 1976), cell-free extracts prepared from frogs' eggs and oocytes have enabled key discoveries in chromatin assembly, DNA replication, cell cycle and other important research fields. When demembranated Xenopus sperm nuclei are incubated in Xenopus egg extracts, the compact sperm chromatin decondenses, origins are 'licensed' for replication and a nuclear envelope reforms. Following this $\sim 20$ min nuclear assembly step, origins are activated, and the DNA is efficiently duplicated in $\sim 30$ min (Blow and Laskey, 1986). This system closely mimics DNA replication cycles of early Xenopus embryos. Furthermore, purified DNA from any source is also assembled into synthetic nuclei and replicated under cell cycle control in this system, albeit less efficiently than sperm nuclei (Blow and Laskey, 1986).

Early studies revealed that replication origins are located at random sequences and are spaced at $\sim 10 \mathrm{~kb}$ intervals in egg extracts and early embryos (for review, (Hyrien et al., 2003)). In

\footnotetext{
Abbreviations used in this paper: EM, electron microscopy; FISH, fluorescent in situ hybridization; FRET, fluorescence-resonance energy transfer; MES, 2-N-morpholinoethanesulphonic acid; NE, nicking endonuclease; RIs, replication intermediates; TCA, trichloroacetic acid; TIRF, total internal reflection fluorescence.
} 
its simplest form, random origin placement implies an exponential distribution of origin separations and therefore a significant proportion of very large inter-origin distances, raising the question of the mechanisms that ensure a reliable and brief replication ending time. To address this question, replication intermediates of large plasmids were examined by electron microscopy (EM), a single molecule technique that allowed precise measurements of replication bubble sizes and spacings. No evidence for spatial regularity in the placement of origins was obtained. However, origins were found to activate throughout $S$ phase at a rate that increased during $S$ phase, allowing to accelerate replication completion late in S phase (Lucas et al., 2000).

To confirm and extend these findings using replicating DNA from sperm nuclei, which is not easily amenable to EM analysis, DNA combing, a technique for stretching and aligning single DNA molecules on a glass coverslip (Michalet etal., 1997), was combined with differential labelling of early- and late-replicated DNA by timed addition of digoxigenin-dUTP and biotin-dUTP to egg extracts (Herrick et al., 2000). Detection of biotin and digoxigenin-labelled replication tracts with fluorescent streptavidin and anti-digoxigenin antibodies on combed DNA molecules allowed to visualize the replication state of large fractions of the Xenopus genome, to precisely measure inter-origin distances and to compute $I(t)$, the rate of initiations per time per length of unreplicated DNA (Herrick et al., 2002, Herrick et al., 2000). I(t) was confirmed to increase during S phase, and mathematical analysis demonstrated that the observed $I(t)$ results in a reproducible replication ending time while minimizing the use of cellular replication proteins (Yang and Bechhoefer, 2008).

DNA combing and other DNA fibre spreading techniques were adapted to yeast and mammalian cells by labelling replication tracts with halogenated thymidine analogs, which, in contrast to tagged dNTPs, can permeate the cytoplasmic membrane (Jackson and Pombo, 1998), and by using a yeast strain expressing a viral thymidine kinase gene (Lengronne et al., 2001). Importantly, the detection of incorporated halogenated thymidine analogs could be combined with FISH with specific DNA probes to identify and orient target DNA molecules (Norio and Schildkraut, 2001). These technical breakthroughs have made DNA combing and related DNA fibre techniques a widely used tool for DNA replication studies. A unique advantage of such single-molecule techniques is that they give access to cell-to-cell variability in DNA replication patterns, whereas cell-population based techniques only provide a population-averaged picture of DNA replication.

There are a number of bottlenecks, however, that currently limit the use of single-molecule replication mapping techniques. First, label detection with antibodies introduces complicated incubations and washing steps during which the DNA molecules tend to detach from the coverslip. Furthermore, the labelled tracts often appear dotty which limits resolution and entails the risk of false negative detection. A second, major bottleneck that prevents the simultaneous analysis of multiple loci is the need of complex FISH probes to identify and orient fibres covering each locus of interest. This further complicates and introduces harsher hybridization/washing steps, severely limiting the total length of genomic regions one can study in a single experiment.

Here we report significant progress in bypassing these two bottlenecks. First, replication tracts can be reliably labelled in Xenopus egg extracts by incorporation of fluorescent dNTPs, eliminating the need for antibody detection. Second, when DNA is stained with
YOYO-1, a fluorescent DNA intercalator, replication bubbles show a distinctly stronger fluorescence than unreplicated segments of the same DNA molecule. Third, DNA barcoding by incorporation of fluorescent dNTPs at specific nicking endonuclease (NE) sites (Chan et al., 2011, Xiao et al., 2007) is compatible with these novel bubble detection techniques, allowing rapid mapping of the combed DNA replication intermediates to their locus of origin.

\section{Experimental Protocols}

\section{Replication of sperm nuclei or $\lambda$ DNA in Xenopus egg extracts}

Interphase egg extracts and demembranated sperm nuclei $\left(6.6 \times 10^{4}\right.$ nuclei/ $\left.\mu \mathrm{L}\right)$ were prepared and frozen as described (Marheineke et al., 2009). Bacteriophage $\lambda$ DNA $(0.5 \mu \mathrm{g} / \mu \mathrm{L})$ was purchased from New England Biolabs. Sperm nuclei or $\lambda$ DNA were incubated at 1,320 nuclei/ $\mu \mathrm{L}$ or $4 \mathrm{ng} / \mu \mathrm{L}$, respectively, in thawed extracts supplemented with an energy regeneration mix [10 u/mL creatine kinase, $10 \mathrm{mM}$ creatine phosphate, $1 \mathrm{mM}$ ATP, $1 \mathrm{mM} \mathrm{MgCl}_{2}$ ], cycloheximide $(250 \mu \mathrm{g} / \mathrm{mL})$, in the presence of 20 $\mu \mathrm{M}$ AlexaFluor647-aha-dUTP (AF647-dUTP) or AlexaFluor555aha-dUTP (AF555-dUTP) (ThermoFisher Scientific), or the same volume of water, at $23^{\circ} \mathrm{C}$.

\section{Quantitation of DNA synthesis}

For quantitation of DNA synthesis by the trichloroacetic acid (TCA) precipitation assay, $0.2 \mu \mathrm{Ci} / \mu \mathrm{L}\left[\alpha^{-32} \mathrm{P}\right]-\mathrm{dATP}$ was added to a final $25 \mu \mathrm{L}$ volume of replication mixture. Timed aliquots $(2.5 \mu \mathrm{L})$ were stopped in $45.5 \mu \mathrm{L}$ of stop mix $(300 \mathrm{mM} \mathrm{NaCl}, 40 \mathrm{mM}$ EDTA $\mathrm{pH} 8,1 \%$ SDS, $0.5 \%$ Triton $\mathrm{X}-100)$. Each aliquot was digested with $2 \mu \mathrm{L}$ of proteinase $\mathrm{K}(10 \mu \mathrm{g} / \mu \mathrm{L}$, Sigma-Aldrich) for at least $1 \mathrm{~h}$ at $55^{\circ} \mathrm{C}$. The digested samples $(50 \mu \mathrm{L})$ were spotted on Whatman GF/C filters and immediately immersed in an ice-cold $5 \% \mathrm{w} / \mathrm{v}$ TCA solution for $20 \mathrm{~min}$. The filters were further washed twice in TCA for $20 \mathrm{~min}$, twice in $100 \%$ ethanol for $5 \mathrm{~min}$, dried onto Whatman paper and radioactivity was measured in a LS6500 Multi-Purpose Scintillation Counter (Beckman Coulter). To measure the total radioactivity input, a $1 \mu \mathrm{L}$ aliquot of the replication mixture was diluted in $47 \mu \mathrm{L}$ of stop mix, digested with proteinase $\mathrm{K}$ as above, and $5 \mu \mathrm{L}$ aliquots were spotted directly onto Whatman GF/C filters and counted as above.

\section{DNA purification}

Replication reactions (50 $\mu \mathrm{L}$ aliquots) were processed for embedding in agarose plugs and DNA purification as described (Marheineke et al., 2009), with the following modifications. Nuclei were pelleted at 2,200 $\mathrm{g}$. The proteinase $\mathrm{K}$ digestion buffer was 100 mM EDTA pH 8, 10 mM Tris- $\mathrm{HCl}$ pH 8, $20 \mathrm{mM} \mathrm{NaCl}, 1 \% \mathrm{~N}-$ Lauroylsarcosine, $2 \mathrm{mg} / \mathrm{mL}$ proteinase $\mathrm{K}$. Plugs were then optionally processed for nick-labelling (see below). In all cases, the final rinsing steps before agarase digestion were in $10 \mathrm{mM}$ Tris- $\mathrm{HCl}$, $1 \mathrm{mM}$ EDTA, pH 6.5. After agarase digestion for $6 \mathrm{~h}$ at $42^{\circ} \mathrm{C}, 2$ more units agarase were added overnight. The following day, 0.8 $\mu \mathrm{L}$ of $100 \mu \mathrm{M}$ YOYO-1 (ThermoFisher Scientific) were added for overnight staining at $42^{\circ} \mathrm{C}$. Agarase was inactivated $30^{\prime}$ at $65^{\circ} \mathrm{C}$ and the samples were diluted in $1.5 \mathrm{~mL}$ of $50 \mathrm{mM} \mathrm{MES} \mathrm{pH} 6.0$ and stored at $4^{\circ} \mathrm{C}$ until DNA combing.

\section{Nick-labelling}

Nick-labelling of purified $\lambda$ DNA was performed by nicking 1.2 
$\mu \mathrm{g}$ of $\lambda$ DNA with $28 u$ of Nt.BspQI nicking endonuclease (New England Biolabs) in $40 \mu \mathrm{L}$ NEBuffer 3.1 for $2 \mathrm{~h}$ at $37^{\circ} \mathrm{C}$, followed by enzyme inactivation for $20 \mathrm{~min}$ at $80^{\circ} \mathrm{C}$. The sample was added with $1 \mathrm{x}$ Thermopol buffer (New England Biolabs), $100 \mathrm{nM}$ dATP $100 \mathrm{nM}$ dCTP, $100 \mathrm{nM}$ dGTP, $100 \mathrm{nM} \mathrm{AF647-dUTP}$ and $4 \mathrm{u}$ of Taq DNA Polymerase (New England Biolabs) in a final volume of $60 \mu \mathrm{L}$ and incubated for $1 \mathrm{~h}$ at $72^{\circ} \mathrm{C}$. The sample was split in two $30 \mu \mathrm{L}$ aliquots and nicks were repaired in a final volume of $50 \mu \mathrm{L}$ containing $1 \mu \mathrm{L}$ PreCR Repair Mix (New England Biolabs), 0.5 $\mathrm{mM} \mathrm{NAD}^{+}$and $100 \mu \mathrm{M}$ of each dNTP for $30 \mathrm{~min}$ at $37^{\circ} \mathrm{C}$. DNA was stained at a 1:10 YOYO-1/base pair ratio for $2 \mathrm{~h}$ at $50^{\circ} \mathrm{C}$, spinned on Illustra Microspin G-50 columns (GE Healthcare) for 2 min at $735 \mathrm{~g}$, diluted in $2.5 \mathrm{~mL}$ of $50 \mathrm{mM}$ MES pH 6.0 and stored at $4^{\circ} \mathrm{C}$ until DNA combing.

For nick-labelling of DNA in agarose plugs after the protease $\mathrm{K}$ digestion step, each plug was washed in $10 \mathrm{mM}$ Tris- $\mathrm{HCl}, \mathrm{pH} 8.0$ and digested with $28 \mathrm{u}$ of $\mathrm{Nt.BspQI}$ as above. After removing the reaction buffer, the plug was molten and the enzyme was heatinactivated for $20 \mathrm{~min}$ at $80^{\circ} \mathrm{C}$. Molten samples were brought to $72^{\circ} \mathrm{C}$ in an Eppendorf Thermotop incubator and nick-translation was performed in the molten agarose in a final volume of $100 \mu \mathrm{L}$ of $1 \times$ Thermopol buffer (New England Biolabs) supplemented with 100 nM dATP, 100 nM dCTP, 100 nM dGTP, 100 nM AF647-dUTP and $4 \mathrm{u}$ of Taq DNA Polymerase (New England Biolabs) for $1 \mathrm{~h}$. The tubes were cooled to $4^{\circ} \mathrm{C}$ until complete solidification of the agarose. Nicks were repaired by incubating the resolidified agarose block $(\sim 100 \mu \mathrm{L})$ in a final $150 \mu \mathrm{L}$ of Thermopol buffer complemented with $1 \mu \mathrm{L}$ PreCR Repair Mix (New England Biolabs), $0.5 \mathrm{mM} \mathrm{NAD}^{+}$ and $100 \mu \mathrm{M}$ of each dNTP for $30 \mathrm{~min}$ at $37^{\circ} \mathrm{C}$. Agarose blocks were rinsed in $10 \mathrm{mM}$ Tris- $\mathrm{HCl}, 1 \mathrm{mM}$ EDTA, $\mathrm{pH} 8.0$ (25 mL per block), once for $10 \mathrm{~min}$ and once for $1 \mathrm{~h}$ on a slowly rotating wheel. A second proteinase $\mathrm{K}$ digestion was performed in $1 \mathrm{~mL}$ per block overnight at $55^{\circ} \mathrm{C}$ followed by final rinses as described in the "DNA purification" section.

\section{DNA combing}

DNA combing was performed on silanized coverslips prepared by plasma cleaning and liquid-phase silanization as described (Labit et al., 2008). Images were acquired on a motorized XY stage of a Nikon Eclipse Ti inverted microscope equipped with a Nikon Plan Apo VC 100x oil immersion objective (N.A. 1.4, W.D. $0.13 \mathrm{~mm}$ ), a QuantEM:512SC EMCCD camera (Photometrics), a X-Cite 120Q illumination source (Lumen Dynamics) and appropriate Semrock fluorescence filter/dichroic mirror sets to prevent any signal leakage between channels. Measurements on each molecule were made using ImageJ and Adobe InDesign and compiled using Microsoft Excel. The data were processed with $\mathrm{R}$ using custom scripts available upon request.

\section{Statistical analysis}

The probability of getting $n$ events in a given segment of the $\lambda$ genome when 198 events occur randomly over the entire $\lambda$ ge-

\section{A}

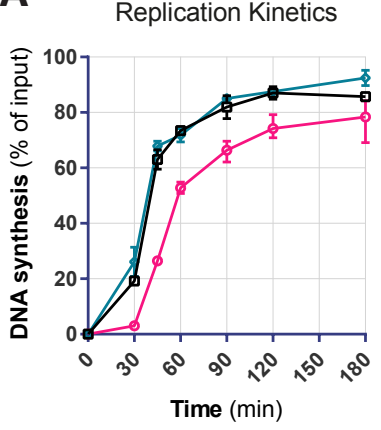

B
C

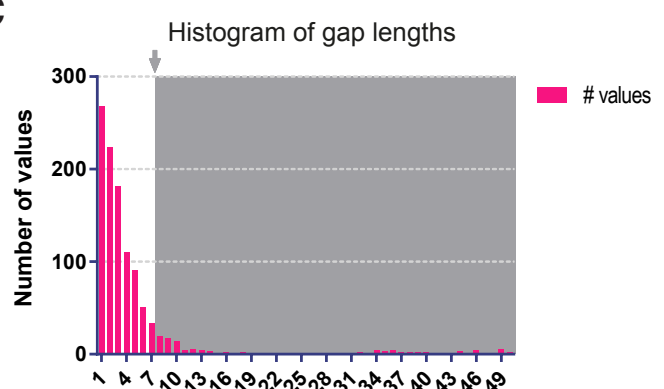

Gap size (pixels)

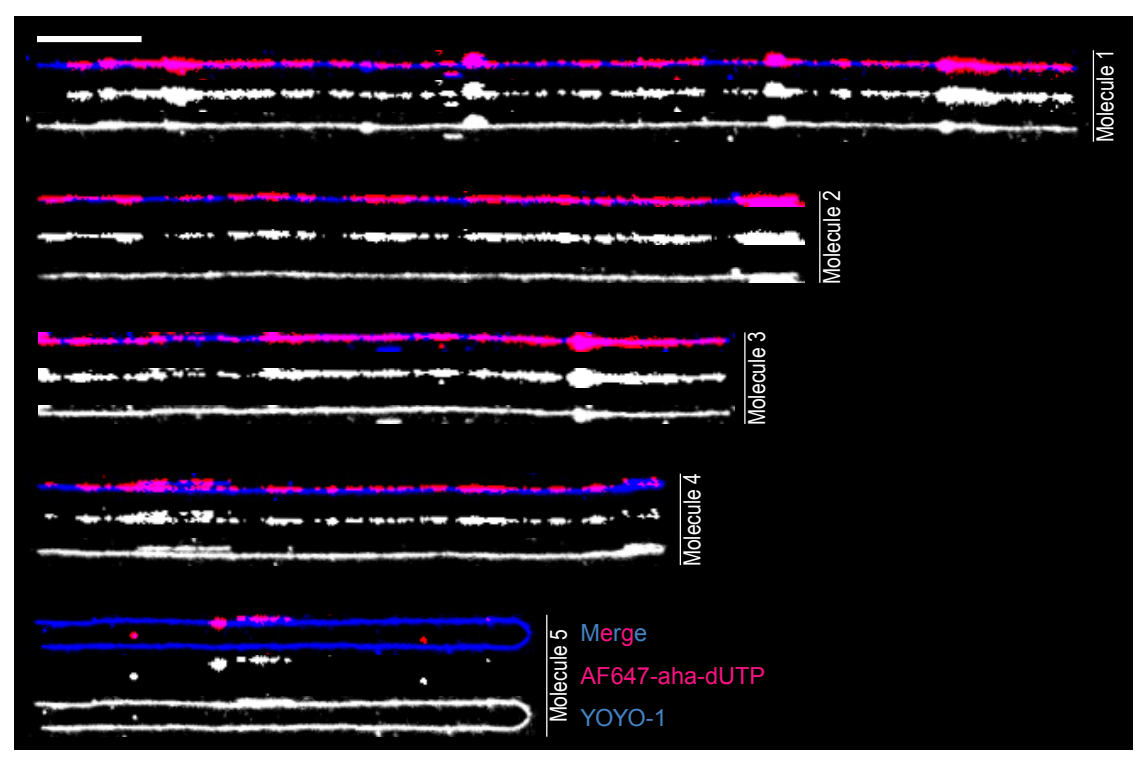

Fig. 1. Labelling replicated DNA with AF647dUTP. (A) Replication kinetics of sperm nucle added to Xenopus egg extracts in the presence of $20 \mu \mathrm{M}$ AF647-dUTP or $20 \mu \mathrm{M}$ dUTP or the same volume of water, as determined by the TCA precipitation assay. The dots and bars show the mean and range of two (dUTP, water) or three (AF647-dUTP) replicates of the experiment with the same egg extract. Similar results were obtained with two independently prepared egg extracts. (B) Combed DNA fibres from sperm nucleiadded to the same egg extract as in $(A)$ in the presence of AF647-dUTP for $120 \mathrm{~min}$. Scale bar, $10 \mu \mathrm{m}(20 \mathrm{~kb})$. Most molecules (examples 1 to 4) were labelled over most of their length but a few large unlabelled stretches (example 5) were also observed. (C) Size distribution (in pixels) of 1,130 unlabelled gaps measured on randomly selected DNA fibres $>10 \mathrm{~kb}$ in length totalling 9,467 kb of DNA from the experiment shown in (B). The distribution was truncated at 50 pixels. The grey arrow indicates the threshold size (7 pixels) below which unlabelled gaps are not considered significant (1 pixel $=0.16$ $\mu \mathrm{m}=0.32 \mathrm{~kb})$. 
nome follows the associated binomial law $\mathrm{B}(198, p)$, where $p$ is the ratio of DNA fibre coverage of the segment to total coverage, as explained in (Letessier et al., 2011). The observed number of initiation events in consecutive $5 \mathrm{~kb}$ segments of the $\lambda$ genome was always included in the corresponding $95 \%$ confidence interval, consistent with the null hypothesis that initiation events are randomly distributed over the $\lambda$ genome.

\section{Results and Discussion}

\section{Incorporation of Alexa Fluor 647-aha-dUTP during DNA rep- lication in Xenopus egg extracts}

Sperm chromatin $(1,320$ nuclei/ $\mu \mathrm{l})$ was incubated in egg extracts in the presence of $\alpha^{32} \mathrm{P}-\mathrm{dATP}$ and $20 \mu \mathrm{M}$ Alexa Fluor 647-ahadUTP (AF647-dUTP) or $20 \mu \mathrm{M}$ dUTP or the same volume of water and replication kinetics was monitored by TCA precipitation (Fig. 1A). Replication started at $30 \mathrm{~min}$ in the control (+ water) extract and reached a plateau at $90 \mathrm{~min}$. The presence of AF647-dUTP only slightly reduced the rate and final amount of DNA replication. This small but reproducible inhibitory effect was not observed with dUTP, suggesting that the fluorochrome moiety of AF647-dUTP was responsible for the inhibition. Similar results were observed with AF555-dUTP (not shown).

To evaluate AF647-dUTP incorporation by DNAcombing, sperm chromatin was replicated in the presence of AF647-dUTP for 120 min, when replication nears completion, DNA was purified, stained with YOYO-1 and combed (Fig. 1B). As expected, fibres were labelled in red (AF647 fluorescence) over most of their length. However, the AF647 signal was not as continuous as the YOYO-1 signal. Several hypotheses may explain the occurrence of unlabelled gaps along the molecules. First, large unlabelled gaps may reflect stretches of unreplicated DNA. Indeed, the TCA assay indicated that only $74 \%$ of the DNA was replicated in this sample. However, if even the smallest gaps represented unreplicated DNA, their frequent occurrence would imply a very high rate of fork stalling and origin activation to account for the high density of red tracts. Results presented below disfavour this hypothesis. Second, the fluorescent moiety of incorporated AF647-dUMP might have been partly removed by DNA repair. To our knowledge, no evidence for such activity has been reported. Third, and most likely, the signal may be quenched by interaction with the combing surface or by photobleaching.

We determined the size distribution of 1,130 unlabelled gaps measured on randomly selected DNA fibres $>10 \mathrm{~kb}$ in length totalling $9,467 \mathrm{~kb}$ of DNA (Fig. 1C). Overall, $65.4 \%$ of the DNA was labelled by AF647, a lower figure than the replication extent of this sample estimated to $74 \%$ by the TCA assay (Fig. 1A). Most gap sizes followed an exponential distribution ranging from 1 to 20 pixels, with a few larger values up to 318 pixels ( 1 pixel $=0.32$ $\mathrm{kb}$ assuming a stretching factor of $2 \mathrm{~kb} / \mu \mathrm{m}$ ). Gaps $\leq 7$ pixels ( $=959$ ) totalled $9.1 \%$ of the DNA. Assuming that such small gaps $(<2.24 \mathrm{~kb})$ actually consist of replicated DNA, the replication extent measured by DNA combing would raise to $74.5 \%$, in excellent agreement with the TCA assay. We therefore suggest that gaps below this $2.24 \mathrm{~kb}$ threshold size are not significant and are due to imperfect signal detection. The resolution of the AF647 labelling technique therefore appears comparable to, if not better than, standard DNA combing (Herrick et al., 2000).

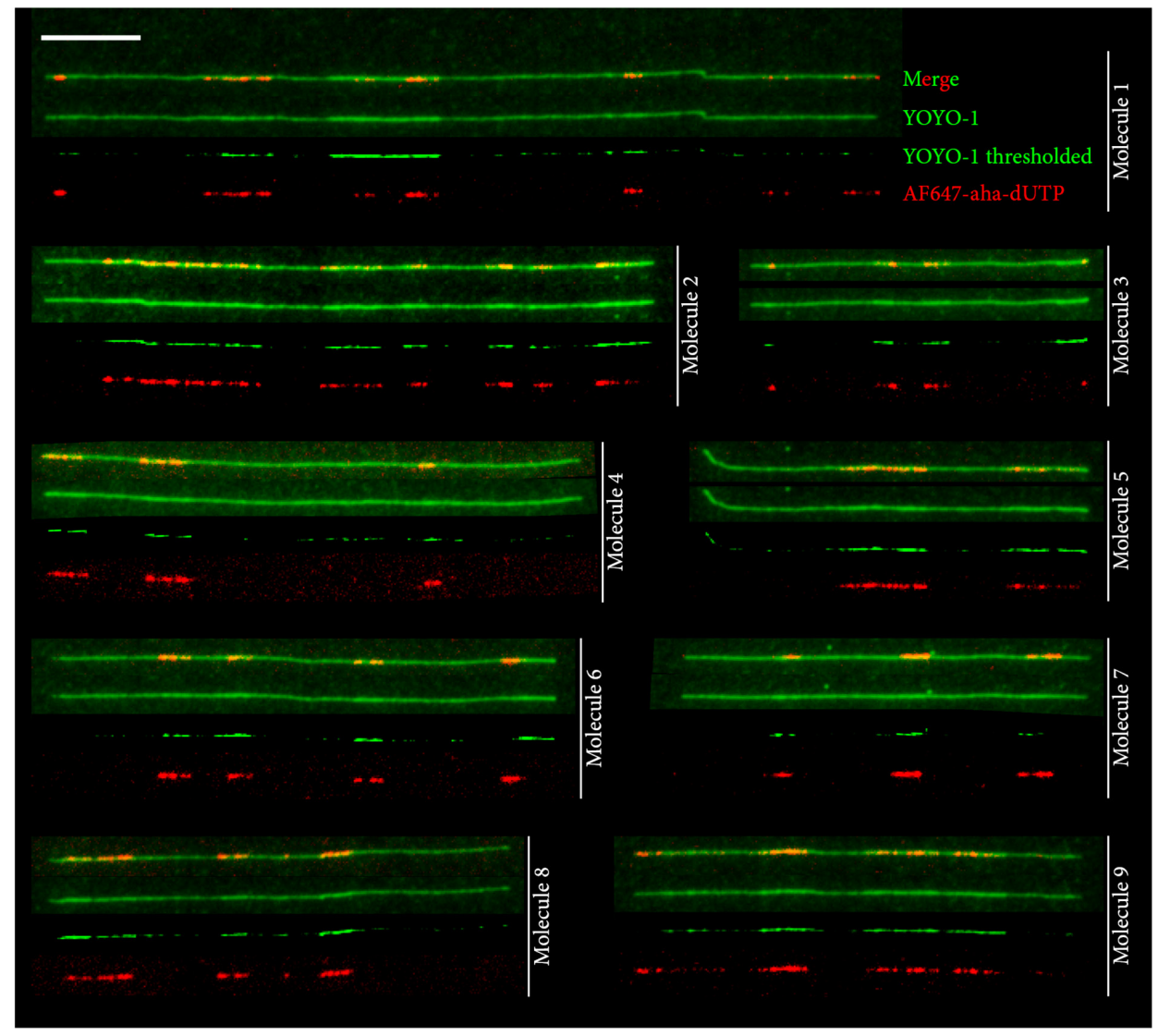

Fig. 2. Visualizing replication bubbles by direct fluorescence of combed DNA. The nine molecules shown are combed DNA replication intermediates from sperm nuclei added to Xenopus egg extracts in the presence of AF647dUTP for $45 \mathrm{~min}$. All the molecules shown come from a single experiment. For each molecule, four images are shown. The green YOYO-1 image reveals the complete DNA molecule. The red AF647-dUTP image reveals the position of replicated segments. The thresholded YOYO-1 image reveals the tracts of strongest YOYO-1 fluorescence, which clearly coincide, at the resolution of the technique, with the AF647labelled tracts. A merge of the raw YOYO-1 and AF647-dUTP images is also shown. Bar, $10 \mu \mathrm{m}(20 \mathrm{~kb})$. 


\section{Two corroborating fluorescent methods for replication bubble detection}

Fig. 2 shows a collection of replicative intermediates extracted after 45 min in egg extracts. Previous EM and DNA combing studies of similar replication intermediates have shown that replicated and unreplicated segments alternate at $\sim 5-50 \mathrm{~kb}$ intervals depending on the local replication extent (Herrick et al., 2000, Lucas et al., 2000). Indeed, when gaps $\leq 7$ pixels were disregarded, the AF647 signal consisted of 5-50 kb stretches, alternating with unlabelled stretches of comparable size. Remarkably, the replicated stretches detected by AF647-dUTP coincided with tracts of stronger YOYO-1 fluorescence. The YOYO1 signal was thresholded using the Brightness/Contrast tools of ImageJ. We could manually define settings that rendered the correlation with the AF647 signal particularly obvious for each molecule.

The increased YOYO-1 fluorescence of replicated segments was observed in four independent experiments. In some experiments, however, the YOYO-1 signal was noisier and less correlated with the replication signal. Further work is required to determine if this is due to variation in surface quality or in the DNA/YOYO-1 ratio during staining.

These results are consistent with experiments by Yardimci et al., (Yardimci etal., 2010), who studied the replication of stretched $\lambda$ DNA molecules in a soluble Xenopusegg extract system in a microfluidic flow cell. These authors showed that tracts of digoxigenin-dUTP incorporated during replication colocalized with tracts of double DNA fluorescence intensity. Our results show that replication bubbles can be detected on combed DNA by two independent yet compatible fluorescent techniques, the increase of YOYO-1 fluorescence and the incorporation of AF647-dUTP. Because the YOYO-1 method does not require metabolic labelling, it should be applicable to detect replication bubbles in DNA from any source.

\section{Barcoding DNA by fluorescent nick-labelling}

A major bottleneck of conventional DNA combing is the need for FISH to identify a genomic locus of interest. This requires a complex collection of probes, hybridization/washing steps, and exhaustive scanning of the coverslips to find the few fibres containing the probed locus. We propose to replace these steps by fluorescent labelling of nicking endonuclease sites, potentially allowing identification of every molecule on the coverslip.

Nicking endonucleases (NEs) are restriction endonucleases which cleave only one of the strands of double-stranded DNA. Xiao et al., (Xiao et al., 2007) have reported that DNA can be labelled with fluorescent dyes at specific sequence motifs by nicking with a NE followed by incorporation of fluorescent ddNTP chain terminators with a DNA polymerase. However, total internal reflection fluorescence (TIRF) microscopy was required to detect the weak labels. A variant reported method consists in incorporating several AF647-dUTPs at each nick site by nick-translation with DNA polymerase I (Chan et al., 2011). In this case, the DNA was stained with YOYO-1 and the nick-translated sites were revealed by fluorescence-resonance energy transfer (FRET) signal from the Alexa fluorophore.

Here, we developed a method that allows detection of nicktranslated sites by standard fluorescent microscopy (Fig. 3; no TIRF, no FRET). Bacteriophage $\lambda$ DNA (48.5 kb) was nicked with Nt.BspQI, nick-translated with AF647-dUTP and Taq DNA

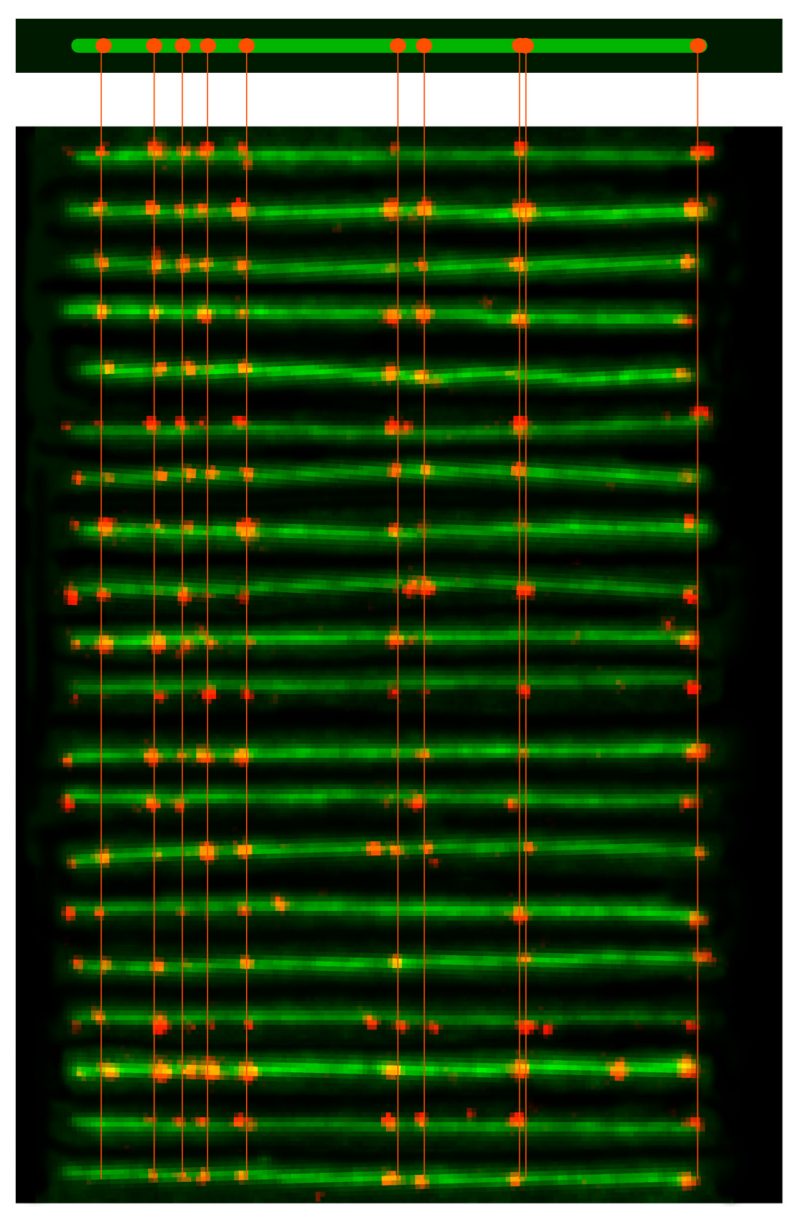

Fig. 3. Nick-labelling of $\lambda$ DNA molecules at Nt.BspQI nicking sites. Top, distribution of Nt.BspQI nicking sites (red dots) along the map of the $\lambda$ genome (green). Bottom, individual combed $\lambda$ DNA molecules (green, YOYO-1 fluorescence) labelled by nick-translation with AF647-dUTP (red) at Nt.BspQI sites. All the molecules shown come from a single experiment. The molecules are aligned under the Nt.BspQl restriction map of $\lambda$ DNA. Vertical red lines indicate alignment. Note the rare occurrence of missing or extra labels. The occasional extra label at the left end is likely due to filling of the 3'-recessed cohesive end, where template sequence limits incorporation to 1 dUTP.

polymerase, which possesses a 5' flap endonuclease activity, and repaired with a PreCR mix. This protocol avoids perturbation of DNA combing by flaps and breakage at single or vicinal nicks. Following DNA combing, we obtained excellent images by standard microscopy (Fig. 3). The resolution (1-2 kb) was good, probably higher than FISH, and almost all (99 out 100) full-length molecules could be unambiguously aligned to the Nt.BspQI map of the $\lambda$ genome, despite the occasional occurrence of missing and extra sites ( $14.1 \%$ and $5.7 \%$ of all sites, respectively). This experiment was reproduced three times with identical results. Therefore, nick-labelling is a suitable method to identify combed DNA molecules on a coverslip.

The method may be further improved if the \% of missing and extra sites could be reduced. We found that extra sites result almost exclusively from background spots present between, as well as over, the DNA molecules. The density of such spots between the molecules was 0.5 spot per area equivalent to a full-length $\lambda$ DNA 
molecule. Given that there are 9 discernable labels on fully labelled molecules, the $\%$ of extra sites due to background would be $0.5 / 9=$ $5.6 \%$, very close to the observed $5.7 \%$. Improved DNA purification from unincorporated AF647-dUTP may reduce this percentage. As to the missing sites, they may be due either to incomplete nicking or, more likely, to quenching by interaction with the combing surface or by photobleaching, just as with replicative tracts. Small gaps in replicative staining due to imperfect signal detection were estimated above to $9.1 \%$ of the DNA. The observed $14.1 \%$ of missing nicking sites is not far above this percentage, suggesting that they may largely be due to the same type of quenching as observed with replicative tracts. If so, there is at present little hope to reduce this percentage below $\sim 10 \%$.

\section{Combining replication bubble and DNA barcode detection}

In our initial attempts to combine bubble and NE site detection, we added $\lambda$ DNA or sperm chromatin to Xenopus egg extracts in the presence of AF555-dUTP, purified the DNA as usual, nick- labelled it with Nt.BspQI and AF647-dUTP, stained it with YOYO-1 and tried to comb it. We repeatedly observed that DNA prepared in this way was not properly stretched during combing. Systematical testing showed that the addition of either Nt.BspQI, Taq polymerase, PreCR mix or their buffers was sufficient to prevent proper stretching of the DNA.

We therefore devised a protocol to clean the nick-labelled DNA while maintaining it in agarose plugs, so as to avoid breakage before combing (see Experimental Protocols). DNA prepared in this way could be combed and labelled NE sites were observed along replicated and unreplicated tracts (Fig. 4). The selected molecules shown on Fig. 4 come from $\lambda$ DNA replicated in egg extracts for $180 \mathrm{~min}$. Replication of $\lambda$ DNA is slower than sperm nuclei, which explains the presence of partially replicated molecules at this time. Linear $\lambda$ DNA molecules added to egg extracts are subjected to active end-joining resulting in the formation of complex concatamers. A few examples of such concatamers are shown on Fig. 4. Segments corresponding to the entirety or specific portions of the

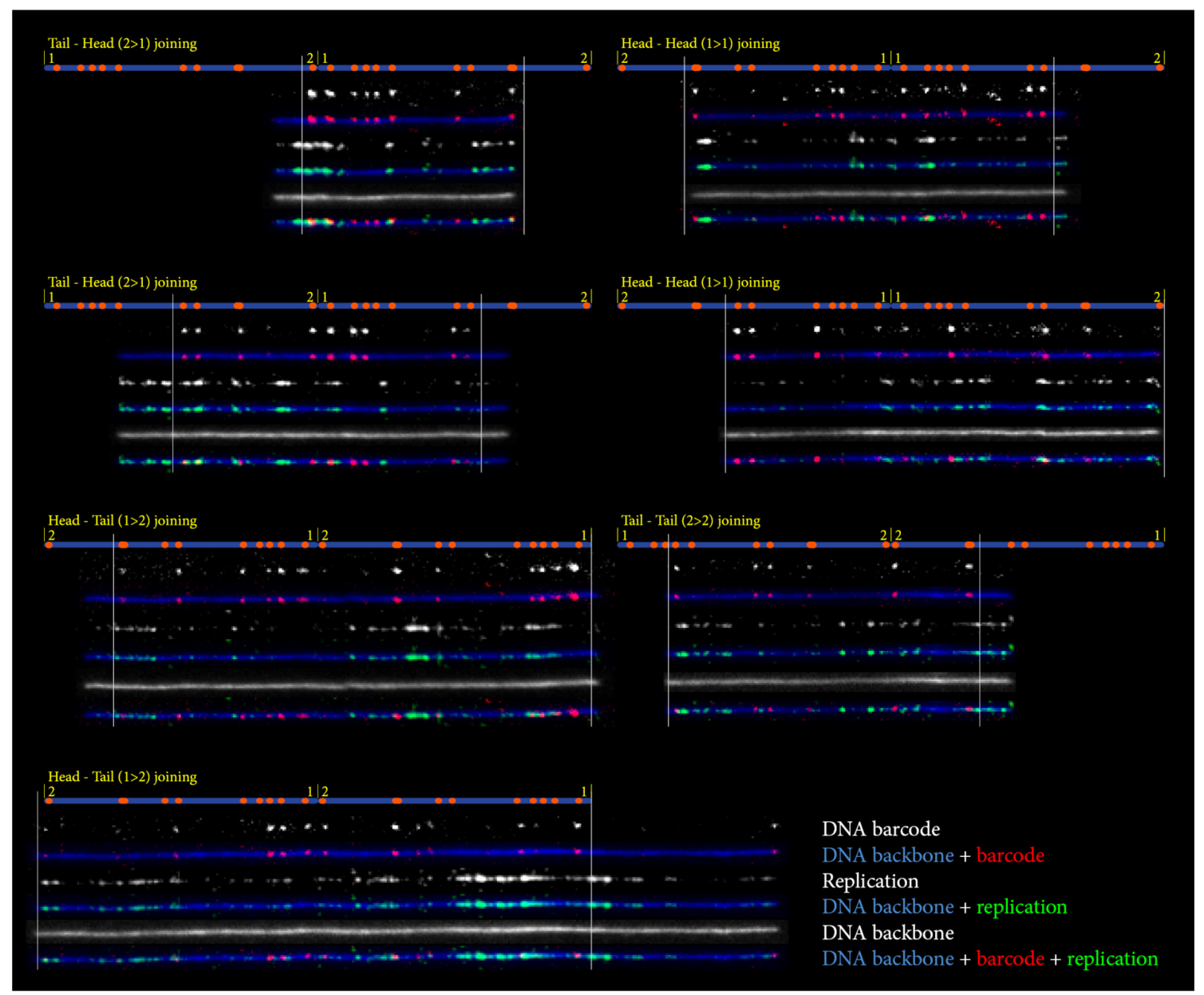

Fig. 4. Combining fluorescent labelling of replication tracks and Nt.BspQl sites on $\lambda$ DNA replicated in Xenopus egg extracts. The molecules shown come from $\lambda$ DNA replicated in Xenopus egg extracts in the presence of AF555-dUTP (green) for 180 min and subsequently labelled by nicktranslation with AF647-dUTP (red) at Nt.BspQI sites. All the molecules shown come from a single experiment. Egg extracts contain end-joining activities that result in the formation of complex concatamers of $\lambda$ DNA. For each of the seven examples shown, the blue line with red dots at the top represents the expected Nt.BspQI map produced by the indicated rearrangement of two $\lambda$ DNA molecules in yellow. The three black-and-white raw images aligned below each map correspond to the observed distribution of Nt.BspQI sites (AF647, top), replication tracts (AF555, middle) and DNA (YOYO-1, bottom) of a single DNA molecule. The three intercalated false-coloured images indicate the merges of YOYO-1 (blue) with AF647 (red) and/or AF555 (green) signals. 
A

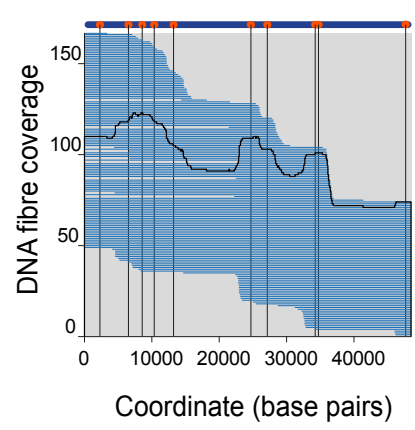

B

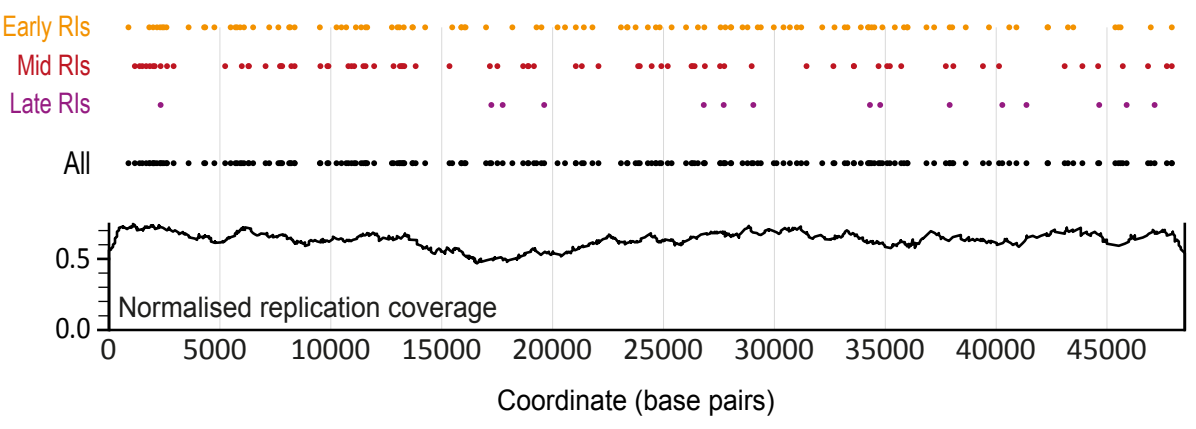

Fig. 5. Homogeneous replication initiation probability of the $\lambda$ DNA sequence. (A) Map position and total DNA coverage of $\lambda$ DNA replication intermediates $(n=167)$ ordered from left to right. All the molecules analysed come from the same experiment as in Figure 4. (B) Position of individual initiation events (midpoint of each replicated segment $<5 \mathrm{~kb}$, excluding fragments overlapping the molecule ends) along the $\lambda$ DNA sequence, for all replication intermediates (RIs) (All RIs, 198 initiations) or for sorted RIs by replication extent (Early R/s, 6-52 \% replication, 55 RIs, 108 initiations; Mid RIs, 53-72 \% replication, 55 R/s, 76 initiations; Late R/s, 73-98 \% replication, 57 R/s, 15 initiations).

$\lambda$ genome could be easily recognized from their barcodes within the concatamers. These results demonstrate that NE barcoding is compatible with fluorescent replicative labelling, allowing to simultaneously reveal both the restriction map and the replication pattern of single DNA molecules using standard fluorescence microscopy, without any FISH or antibody detection step. This experiment was performed twice with similar results.

We used data from a total of $167 \lambda$ DNA molecules from the experiment shown in Fig. 4 to look for any inhomogeneity in replication initiation probability along the $\lambda$ DNA sequence. The position of each molecule and the resulting local coverage are indicated on Fig. 5A. Not unexpectedly, the coverage was slightly higher near Nt.BspQI sites. We plotted the location of all replication tracts (filtered for gaps $\leq 7$ pixels) and computed the replication coverage (normalized to total coverage) along the $\lambda$ DNA sequence (Fig. 5B, bottom). The normalized replication coverage was fairly homogeneous, except perhaps for a small dip over map position 15,000 to 20,000 . To determine the location of replication initiation events, we determined the midpoint of all replicated segments $<5 \mathrm{~kb}$, excluding segments overlapping the molecule ends. This size cut-off minimizes the possibility that the replicated segment arises from merging of two or more replication bubbles. We then plotted the midpoint of each segment $(n=$ 198) along the $\lambda$ DNA sequence for all replication intermediates or for replication intermediates sorted by replication extent into three equally populated classes (early-, mid- and late-replicating intermediates) (Fig. 5B, top). The density of initiation events appeared fairly homogeneous along the DNA sequence for the three classes of replication intermediates. Statistical testing (see Experimental Protocols) confirmed that, at this coverage, the probability of replication initiation is spatially homogeneous over the $\lambda$ genome in Xenopus egg extracts.

\section{Acknowledgments}

We thank Malik Kahli for help with egg extracts and Benoit Le Tallec and Arach Goldar for helpful discussions. This work was supported by the Ligue Nationale contre le Cancer (Comite de Paris), the Fondation $A R C$ pour la recherche sur le cancer and the Agence Nationale pour la Recherche (ANR-15-CE12-0011-01). FDC was supported by a Ph-D fellowship from the Université Pierre et Marie Curie.

\section{References}

BLOW, J.J. and LASKEY, R.A. (1986). Initiation of DNA replication in nuclei and purified DNA by a cell-free extract of Xenopus eggs. Cell 47: 577-587.

CHAN, S.H., STODDARD, B.L. and XU, S.Y. (2011). Natural and engineered nicking endonucleases--from cleavage mechanism to engineering of strand-specificity. Nucleic Acids Res 39: 1-18.

GANDINI ATTARDI, D., MARTINI, G., MATTOCCIA, E. and TOCCHINI-VALENTINI, G.P. (1976). Effect of Xenopus laevis oocyte extract on supercoiled simian virus 40 DNA: formation of complex DNA. Proc Natl Acad Sci USA 73: 554-558.

HERRICK, J., JUN, S., BECHHOEFER, J. and BENSIMON, A. (2002). Kinetic model of DNA replication in eukaryotic organisms. $J$ Mol Biol 320: 741-750.

HERRICK, J., STANISLAWSKI, P., HYRIEN, O. and BENSIMON, A. (2000). Replication Fork Density Increases During DNA Synthesis in X. laevis Egg Extracts. J Mol Biol 300: 1133-1142.

HYRIEN, O., MARHEINEKE, K. and GOLDAR, A. (2003). Paradoxes of eukaryotic DNA replication: MCM proteins and the random completion problem. Bioessays 25: 116-125.

JACKSON, D.A. and POMBO, A. (1998). Replicon clusters are stable units of chromosome structure: evidence that nuclear organization contributes to the efficient activation and propagation of S phase in human cells. J Cell Biol 140: 1285-1295.

LABIT, H., GOLDAR, A., GUILBAUD, G., DOUARCHE, C., HYRIEN, O. and MARHEINEKE, K. (2008). An optimized easy method for preparing silanized surfaces for FISH and replication mapping on combed DNA fibers. BioTechniques 45:649-658.

LENGRONNE, A., PASERO, P., BENSIMON, A. and SCHWOB, E. (2001). Monitoring $S$ phase progression globally and locally using BrdU incorporation in TK(+) yeast strains. Nucleic Acids Res 29: 1433-1442.

LETESSIER, A., MILLOT, G.A., KOUNDRIOUKOFF, S., LACHAGES, A.M., VOGT, N., HANSEN, R.S., MALFOY, B., BRISON, O. and DEBATISSE, M. (2011). Celltype-specific replication initiation programs set fragility of the FRA3B fragile site. Nature 470: 120-123.

LUCAS, I., CHEVRIER-MILLER, M., SOGO, J.M. and HYRIEN, O. (2000). Mechanisms Ensuring Rapid and Complete DNA Replication Despite Random Initiation in Xenopus Early Embryos. J Mol Biol 296: 769-786.

MARHEINEKE, K., GOLDAR, A., KRUDE, T. and HYRIEN, O. (2009). Use of DNA combing to study DNA replication in Xenopus and human cell-free systems In DNA Replication Methods and Protocols., (ed. Z.DALGAARD, S. V. A. J.). Springer, New York, pp. 575-603.

MATTOCCIA, E., ATTARDI, D.G. and TOCCHINI-VALENTINI, G.P. (1976). DNArelaxing activity and endonuclease activity in Xenopus laevis oocytes. Proc Natl Acad Sci USA 73: 4551-4554.

MICHALET, X., EKONG, R., FOUGEROUSSE, F., ROUSSEAUX, S., SCHURRA, C. HORNIGOLD, N., VAN SLEGTENHORST, M., WOLFE, J., POVEY, S., BECKMANN, J.S. et al., (1997). Dynamic molecular combing: stretching the whole human genome for high- resolution studies. Science 277: 1518-1523. 
NORIO, P. and SCHILDKRAUT, C.L. (2001). Visualization of DNA replication on individual Epstein-Barr virus episomes. Science 294: 2361-2364.

XIAO, M., PHONG, A., HA, C., CHAN, T.F., CAI, D., LEUNG, L., WAN, E., KISTLER, A.L., DERISI, J.L., SELVIN, P.R. et al., (2007). Rapid DNA mapping by fluorescent single molecule detection. Nucleic Acids Res 35: e16.
YANG, S.C. and BECHHOEFER, J. (2008). How Xenopus laevis embryos replicate reliably: Investigating the random-completion problem. Phys Rev E Stat Nonlin Soft Matter Phys 78: 041917.

YARDIMCI, H., LOVELAND, A.B., HABUCHI, S., VAN OIJEN, A.M. and WALTER, J.C. (2010). Uncoupling of sister replisomes during eukaryotic DNA replication. Mol Cell 40: 834-840. 


\section{Further Related Reading, published previously in the Int. J. Dev. Biol.}

Control of timing of embryonic M-phase entry and exit is differentially sensitive to CDK1 and PP2A balance Mohammed El Dika, Damian Dudka, Claude Prigent, Jean-Pierre Tassan, Malgorzata Kloc and Jacek Z. Kubiak Int. J. Dev. Biol. (2014) 58: 767-774

Identification of the sperm motility-initiating substance in the newt, Cynops pyrrhogaster, and its possible relationship with the acrosome reaction during internal fertilization

Toshihiko Watanabe, Hideo Kubo, Shinya Takeshima, Mami Nakagawa, Manami Ohta, Saori Kamimura, Eriko Takayama-Watanabe, Akihiko Watanabe, and Kazuo Onitake

Int. J. Dev. Biol. (2010) 54: 591-597

Signalling molecules involved in mouse bladder smooth muscle cellular differentiation

Benchun Liu, Dongxiao Feng, Guiting Lin, Mei Cao, Yuet Wai Kan, Gerald R. Cunha and Laurence S. Baskin Int. J. Dev. Biol. (2010) 54: 175-180

Nuclear reprogramming in zygotes

Chanchao Lorthongpanich, Davor Solter and Chin Yan Lim

Int. J. Dev. Biol. (2010) 54: 1631-1640

Faithful reprogramming to pluripotency in mammals - what does nuclear transfer teach us? Julien Maruotti, Alice Jouneau and Jean-Paul Renard Int. J. Dev. Biol. (2010) 54: 1609-1621

Gonad-stimulating substance-like molecule from the radial nerve of the sea cucumber Hideki Katow, Tomoko Katow and Akihiko Moriyama

Int. J. Dev. Biol. (2009) 53: 483-491

Cyclin B2/cyclin-dependent kinase1 dissociation precedes CDK1 Thr-161 dephosphorylation upon M-phase promoting factor inactivation in Xenopus laevis cell-free extract

Franck Chesnel, Franck Bazile, Aude Pascal and Jacek Z. Kubiak

Int. J. Dev. Biol. (2007) 51: 297-305

Remodeling of sperm chromatin induced in egg extracts of amphibians.

C Katagiri and K Ohsumi

Int. J. Dev. Biol. (1994) 38: 209-216

The influence of mouse sera, regenerating liver extracts and bacterial products on the abilities of different cells in vitro.

N Zarkovic, M Osmak, D Novak, N Lers and M Jurin

Int. J. Dev. Biol. (1991) 35: 239-249

Hormonal factors from the mammalian pineal gland interfere with cell development in Hydra.

W A Müller, C Bartsch, H Bartsch, I Maidonis and E Bayer

Int. J. Dev. Biol. (1998) 42: 821-824
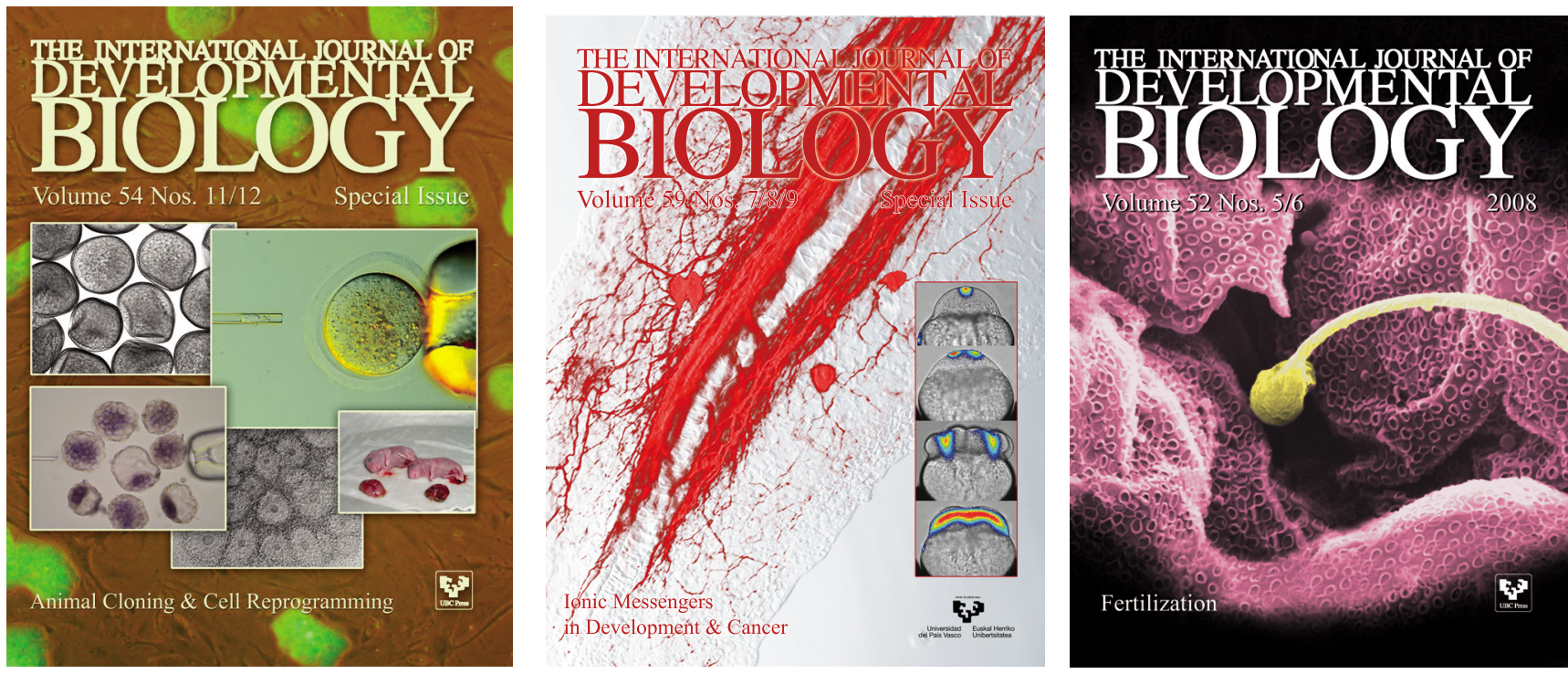